\title{
Lite Maker: Um Fab Lab Móvel para Aplicação de Atividades Mão na Massa com Estudantes do Ensino Básico
}

\author{
André L. M. Santana ${ }^{1}$, André L. A. Raabe ${ }^{12}$, Luís F. M. Santana ${ }^{1}$, Marli F. Vick \\ Vieira $^{12}$, Gustavo L. Ramos ${ }^{1}$, Aldo A. dos Santos ${ }^{1}$ \\ ${ }^{1}$ Laboratório de Inoavação Tecnológica na Educação (LITE) \\ ${ }^{2}$ Programa de Pós-Graduação em Educação \\ Universidade do Vale de Itajaí (UNIVALI) \\ Itajaí - SC - Brazil \\ \{andrelmseunivali.br, raabe@univali.br\}
}

\begin{abstract}
This paper reports the experience of hands-on activities - makers developed with sixteen (16) students from Primary School Yolanda Laurindo Ardigó - Itajaí. For the purposes of this activity was sort build a kind of mobile fab lab, called Lite Maker. Students of primary school were encouraged to create different products based on the concepts learned during their experience at school and in the contents assimilated the stations offered by Lite Maker. The activities took place in (3) three day, on the first day the students learned about the existing tools in the Lite Makers, on the second organized themselves into teams and started its projects, On the last day finished their projects and presented to those involved. The results indicate that makers activities in a mobile lab fab are valid, it stimulates curiosity, interaction, inventiveness, collaboration and make the players participating in the creation process. The involvement of the school community was a positive Question to the participation of students and application of the project.
\end{abstract}

Resumo. $O$ artigo relata a experiência de atividades mãos na massa-makers - desenvolvidas com 16 (dezesseis) estudantes da Escola Básica Yolanda Laurindo Ardigó da cidade de Itajaí. Para aplicação desta atividade foi construído uma espécie de fab lab móvel, chamado de Lite Maker. Os estudantes do ensino básico foram estimulados a criar produtos diferentes, com base nos conceitos apreendidos durante sua vivência na escola e nos conteúdos assimilados nas estações ofertadas pelo Lite Maker. As atividades aconteceram em 3 (três) dias alternados, no primeiro dia os estudantes aprenderam sobre as ferramentas existentes no Lite Maker, no segundo organizaram-se em equipes e iniciaram seus projetos, no último dia finalizaram os projetos e apresentaram para os envolvidos. Os resultados indicam que atividades makers em um fab lab móvel são positivas para formação de um estudante, pois estimulam a curiosidade, a interação, a inventividade, a colaboração e tornam os participantes protagonistas do 
V Congresso Brasileiro de Informática na Educação (CBIE 2016)

Anais do XXII Workshop de Informática na Escola (WIE 2016)

processo de criação. O engajamento da comunidade escolar foi um quesito positivo à participação dos estudantes e aplicação do projeto.

\section{Introdução}

Cada ser humano possui características particulares, que o torna diferente de qualquer outro individuo na forma de pensar, agir, criar e principalmente na forma de descobrir. Tais características, aprimoradas e desenvolvidas com base na cultura e convivência, influenciam em diferentes aspectos da vida cotidiana de um indivíduo, que necessita de abordagens distintas para instigar e aprimorar habilidades cognitivas.

A abordagem construcionista (PAPERT, 1980) implica no envolvimento do indivíduo como protagonista no processo de aprendizagem, a construção do conhecimento desse educando ocorre com base na efetivação de uma ação concreta, que tem como resultado um produto palpável desenvolvido com uso de tecnologias e que precisa ser de interesse de quem o produz.

$\mathrm{O}$ uso de tecnologias permite desenvolver o pensamento computacional no estudante, que por meio de erros e acertos expande sua capacidade de resolução de problemas em diferentes níveis de complexidade (BERS et al., 2014).

O termo pensamento computacional para Blikstein (2013) consiste na aprendizagem prática, que permite ao estudante praticar conceitos estudados em sala de aula através de experimentos mão na massa, buscando proporcionar ao sujeito que o desenvolve uma maneira de utilizar a tecnologia como ferramenta catalisadora para solução de problemas. Tanto a aprendizagem prática quanto o pensamento computacional convergem para um ensino que prioriza a criatividade, inventividade e produtividade dos estudantes, que são protagonistas no processo do desenvolvimento de seu próprio conhecimento.

Santana (2015) trata o pensamento computacional como uma ponte metodológica, que interliga as áreas de Engenharia e Ciência através da organização do modo de pensar, planejar e construir. Os conceitos apresentados por Santana e Blikstein tratam a aprendizagem prática, através de experimentação como uma ferramenta capaz de catalisar o potencial de solucionar problemas.

A aprendizagem prática e o pensamento computacional tratam o estudante como protagonista do processo de construção do seu conhecimento, valorizando a experiência. Tratamento que permite ao estudante aprender com seus erros, acertos e com a satisfação em compreender assuntos do seu próprio interesse, indícios de raízes na cultura maker. (BLIKSTEIN, 2009).

Para o desenvolvimento de habilidades inventivas e criativas Blikstein (2013, 2008) indica a necessidade de ambientes com características específicas como é o caso dos fab labs - Laboratórios de Fabricação - que são ambientes makers. Um fab lab possui um propósito inovador, que reúne um conjunto de tecnologias ${ }^{1}$ digitais e fisicas, ferramentas eletrônicas entre outros artefatos que permitem trabalhar no desenvolvimento de projetos de forma criativa, colaborativa e cooperativa. Milne,

\footnotetext{
${ }^{1} \mathrm{Na}$ pesquisa realizada por Bombasar et al. (2015) destacam-se tecnologias que são utilizadas para estimular o pensamento computacional.
} 
V Congresso Brasileiro de Informática na Educação (CBIE 2016)

Anais do XXII Workshop de Informática na Escola (WIE 2016)

Riecke e Antle (2014) definem o indivíduo maker como uma pessoa qualquer que tenha interesse em combinar tecnologia, conhecimento e computação.

A experiência relatada nesse artigo teve como objetivo promover o pensamento computacional de forma criativa e inovadora, numa perspectiva construcionista baseada no aprendizado prático, com raízes na cultura maker, que oportuniza aos participantes criar sua própria tecnologia concretizando projetos pessoais, suprindo suas necessidades em um ambiente inovador.

Para alcançar estes objetivos e auxiliar outras instituições no mesmo processo, foi projetado o Lite Maker, que consiste em um fab lab móvel que contém rodas, capaz de abordar uma série de atividades práticas em qualquer lugar. Nas próximas seções deste trabalho serão apresentados os elementos principais deste carrinho, a metodologia utilizada para aplicação do mesmo, os principais resultados e conclusões acerca deste relato de experiência. As atividades ocorreram em uma escola da rede pública de ensino com estudantes do $7^{\circ}$ ao $9^{\circ}$ ano do ensino básico.

\section{Materiais e Métodos}

O Lite, Laboratório de Inovação Tecnológica na Educação, é um laboratório que está crescendo e aprimorando-se para apoiar e fomentar a cultura maker em projetos desenvolvidos pelos seus colaboradores e estudantes.

Integrantes do Lite criaram o Lite Maker, nome do carrinho móvel construído para aplicação dessa pesquisa, e inspiram-se no trabalho de McKay e Peppler (2013). Para esses pesquisadores a criação de um móvel capaz de levar atividades criativas e práticas para as escolas pode despertar educandos para um novo modo de ensino, que fomente a cultura da criação mão na massa tornando o estudante protagonista no processo de criação de produtos de seu interesse.

O Lite Maker conta com 4 (quatro) estações diferentes para trabalhos in loco. Necessita como infraestrutura uma saída de energia (tomada) de $220 \mathrm{v}$, pois o projeto consta com adaptadores e extensões para a conexão de todos os equipamentos necessários para as atividades planejadas. É necessária ainda a internet WI-FI para acesso a repositórios de conteúdos $3 \mathrm{D}$ e acesso a conteúdos de inspiração para os projetos a serem desenvolvidos. Cada bancada tem seu papel no projeto dos estudantes, não sendo exclusivo ou obrigatório o uso de todas as estações. O projetista estudante, pode vivenciar experiências que contribuam para a concretização de seu trabalho, desenvolvendo algo de seu interesse e com ferramentas que viabilizem a implementação de um trabalho singular e dinâmico.

As estações podem ser caracterizadas conforme o Quadro 1, nas quais é possível identificar o material disponibilizado para os grupos de estudantes utilizá-los no contexto desta pesquisa.

\begin{tabular}{|l|l|}
\hline $\begin{array}{l}\text { Bancada de } \\
\text { Marcenaria }\end{array}$ & $\begin{array}{l}\text { Nesta bancada os materiais utilizados são ferramentas de marcenaria como: furadeira; martelo; lixa; serra; } \\
\text { conjunto de chaves Philips e Fenda; alicates; pregos e parafusos; esquadros; pequenas gabaritos e } \\
\text { chapas/peças de madeirapara construção. } \\
\text { Os estudantes aprendem sobre medidas, escalas, uso das ferramentas e atividades de construção v oltadas a } \\
\text { comportamentos mecânicos. }\end{array}$ \\
\hline $\begin{array}{l}\text { Bancada de } \\
\text { Decoração }\end{array}$ & $\begin{array}{l}\text { Os materiais utilizados consistem em: plotter de corte; objetos de scrapbook; materiais de corte e reciclados; } \\
\text { barbantes; palitos de picolé; papéis coloridos; enfeites; colas; fitas adesivas; cordões; botões; laços; canetas; } \\
\text { esquadros e grampeadores. }\end{array}$ \\
\hline
\end{tabular}


V Congresso Brasileiro de Informática na Educação (CBIE 2016)

Anais do XXII Workshop de Informática na Escola (WIE 2016)

\begin{tabular}{|l|l|}
\hline & $\begin{array}{l}\text { Estes materiais contribuem para a concepção estética dos projetos, estimulando o lado criativo de cada grupo } \\
\text { de trabalho ou estudante. }\end{array}$ \\
\hline $\begin{array}{l}\text { Bancada de } \\
\text { Prototipação 3D }\end{array}$ & $\begin{array}{l}\text { Esta bancada consta com materiais voltados para a prototipação rápida com impressora 3D. É utiliza do um } \\
\text { notebook para busca em repositórios e desenho dos estudant es, bobinas de filamentos de material de } \\
\text { impressão (PLA) e uma impressora 3D. } \\
\text { Com esses materiais a bancada tem como objetivo ensinara prototipação rápida de pequenos objetos em 3D } \\
\text { e a impressão do mesmo, exercendo uma at ividade que contribui com noções de espacialida de, visão 3 D, } \\
\text { dimensionamento e escala, além de estimular a criatividade e apresentar uma ferramenta que não faz parte } \\
\text { do contexto das escolas públicas brasileiras. }\end{array}$ \\
\hline $\begin{array}{l}\text { Bancada de } \\
\text { Eletrônica }\end{array}$ & $\begin{array}{l}\text { Conta com materiais como: protoboard; fios M X F; leds de várias cores; ferros de solda; resistores; } \\
\text { O objetivo desta bancada consiste em ensinar conceitos de física, com base na prática do funcionamento de } \\
\text { componentes, demonstrando aos estudantes que eles possuem capacidade de criar projetos que se enquadrem } \\
\text { em seus cotidianos. }\end{array}$ \\
\hline
\end{tabular}

Quadro 1. Descrição das bancadas de trabalho do Lite Maker.

Para construção do Lite maker foi necessário o levantamento dos materiais especificados no Quadro 2. Estes materiais foram selecionados com o objetivo de proporcionar a outras instituições a possibilidade de implementar um carrinho Maker de baixo custo, uma vez que o projeto foi especificado para ser de código aberto e com informações públicas.

\begin{tabular}{|c|l|l|}
\hline \multicolumn{3}{|c|}{ MATERIAIS NECESSÁRIOS PARA A CONSTRUÇÃO DO LITE MAKER ${ }^{2}$} \\
\hline QUANTIDADE & \multicolumn{1}{|c|}{ DESCRIÇÃO } & \multicolumn{1}{c|}{ MEDIDAS } \\
\hline 02 & Chapa lateral em MDF & $560 \times 820 \mathrm{~mm}$ \\
\hline 01 & Chapa Central em MDF & $560 \times 820 \mathrm{~mm}$ \\
\hline 01 & Chapa Interior em MDF & $774 \times 578 \mathrm{~mm}$ \\
\hline 01 & Chapa Tampo Superior MDF & $810 \times 578 \mathrm{~mm}$ \\
\hline 01 & Chapa Fundo MDF & $774 \times 820 \mathrm{~mm}$ \\
\hline 02 & Prateleiras em MDF & $560 \times 360 \mathrm{~mm}$ \\
\hline 02 & Chapas de suporte em MDF & $578 \times 100 \mathrm{~mm}$ \\
\hline 04 & Caixas plásticas (estilo supermercado) & $300 \times 360 \times 550 \mathrm{~mm}$ \\
\hline 02 & Rodas fixas & $120 \mathrm{~mm}$ \\
\hline 02 & Rodas móveis com freio & $120 \mathrm{~mm}$ \\
\hline 01 & Barra de apoio de metal & $700 \mathrm{~mm}$ \\
\hline
\end{tabular}

Quadro 2. Relação de materiais para construção do Carrinho.

O projeto teve por objetivo estimular a cultura de aprendizagem prática e apoiar a replicação de outros carrinhos. Nas próximas seções serão apresentados os resultados da aplicação das atividades mão na massa com o uso de um fab lab móvel, o Lite Maker, em uma escola básica da rede pública de Itajaí.

\section{Relato de Experiência}

A dinâmica ocorreu durante 3 (três) dias, 30 de novembro, 02 e 05 de dezembro de 2015, na Escola Básica Yolanda Laurindo Ardigó $^{3}$ situada na cidade de Itajaí com 16

\footnotetext{
2 Detalhes do projeto podem ser encontrados em $<$ http://lite.acad.univali.br $>$

${ }^{3}$ Maiores informações disponível em: $<$ http://escolayolandaardigo.blogspot.com.br/>
} 
V Congresso Brasileiro de Informática na Educação (CBIE 2016)

Anais do XXII Workshop de Informática na Escola (WIE 2016)

(dezesseis) estudantes do ensino fundamental, que foram pré-selecionados pela própria escola.

A utilização do Lite Maker envolveu uma dinâmica de 3 (três) dias de trabalho, durante 4 (quatro) horas por dia, com um intervalo 15 (quinze) minutos após 2 (duas) horas de atividades, totalizando 12 (doze) horas de atividades. Esta dinâmica teve o envolvimento de 8 (oito) integrantes do Lite, contando com professores e bolsistas colaboradores. A atividade ocorreu em uma sala de aula cedida pela própria escola e que foi organizada conforme o layout na Figura 1. No primeiro dia da aplicação da atividade foi realizada a introdução do projeto, sendo apresentada sua finalidade e também avaliada as expectativas dos estudantes.

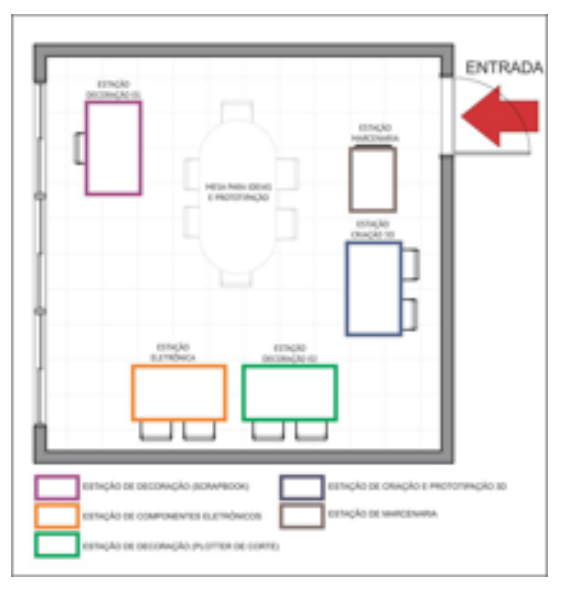

Figura 1 - Layout do local da aplicação da atividade

Após a apresentação inicial, os estudantes organizaram-se em 4 (quatro) equipes não ocorrendo restrição ou sorteio. Os grupos de estudantes iniciaram um rodízio para conhecer as bancadas de trabalho e avaliar seu potencial. Cada grupo teve 1 (uma) hora de explicação sobre o conteúdo que integra cada bancada, informações que foram explanadas por 1 (um) ou 2 (dois) colaboradores do Lite, facilitadores responsáveis pelas bancadas do Lite Maker:

- Bancada prototipação rápida e modelagem 3D (Figura 2). Todas as equipes receberam um breve conceito referente a modelagem 3D (Figura 3), desenvolveram formas geométricas e as manipularam (soma, subtração, escala, intersecção entre objetos). Tiveram contato com o software Sketchup ${ }^{4}$ que realiza as operações executadas. Além da modelagem 3D, os estudantes receberam instruções de como funcionava a impressora 3D, o seu material de trabalho (PLA - ácido poliático) e como efetuar a inserção e remoção deste material. Todas as equipes tiveram a oportunidade de remeter um modelo para impressão na impressora 3D, este modelo foi escolhido no repositório ${ }^{5}$ da própria Makerbot ${ }^{6}$ (fabricante da impressora). A necessidade da busca no repositório da própria Makerbot fez-se necessário para que o modelo escolhido passasse pelo menor número de alterações em software, otimizando assim o tempo da impressão. Os estudantes foram instruídos a buscar

\footnotetext{
${ }^{4}$ www.sketchup.com

${ }^{5}$ www.thingverse.com

${ }^{6}$ www.makerbot.com
} 
V Congresso Brasileiro de Informática na Educação (CBIE 2016)

Anais do XXII Workshop de Informática na Escola (WIE 2016)

modelos que não ultrapassassem 40 minutos de impressão, sendo assim, todas as esquipes tiveram a oportunidade de passar pela mesma experiência.

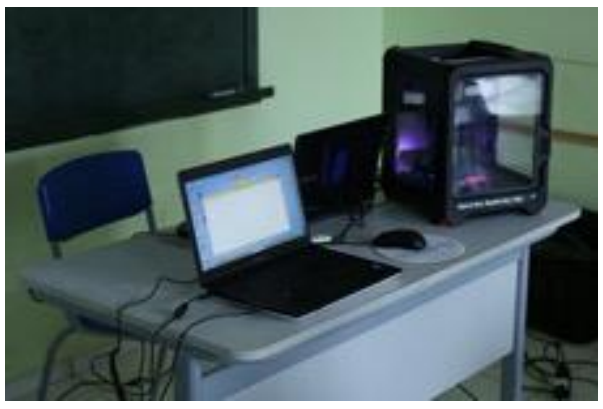

Figura 2 - Bancada de prototipagem rápida

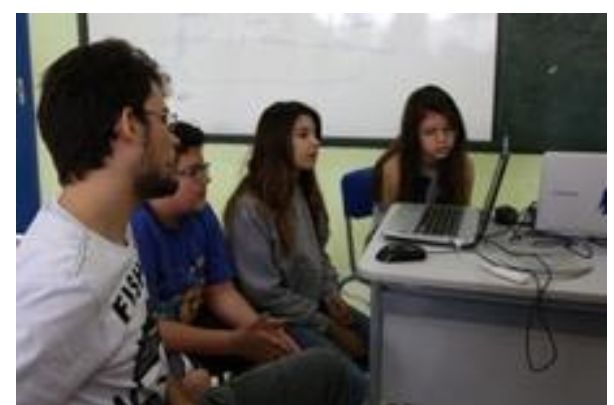

Figura 3 - Estudantes modelando em 3D

- Bancada da Marcenaria. Para esta bancada o próprio Lite Maker foi utilizado como base, os estudantes receberam informações sobre as ferramentas disponíveis na bancada, conceitos de acabamento com as lixas de diferentes gramaturas, técnicas para o uso das serras e uma explicação sobre os tipos de madeiras. Além de obter informações sobre os conceitos das ferramentas de marcenaria, os estudantes também executaram as tarefas de corte, lixa e colagem da madeira, tudo com a supervisão de um responsável.

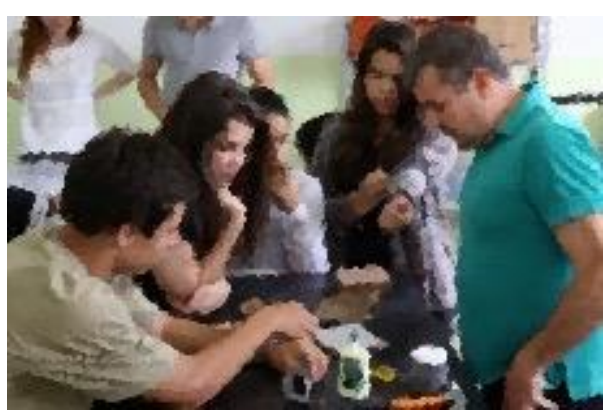

Figura 4 - Estudantes recebendo instruções

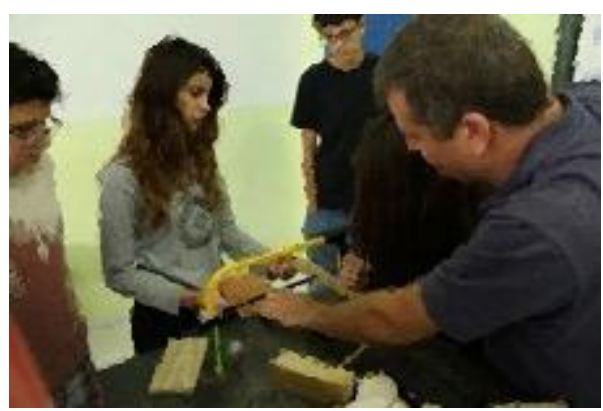

Figura 5 - Estudantes executando tarefas

- Bancada da Eletrônica. Os estudantes foram apresentados ao mundo da eletrônica. Conceitos de polaridade, circuitos, voltagem e medição preencheram à tarde dos estudantes nesta bancada. Todos puderam executar experimentos como a criação de circuitos, solda e manuseio do multímetro, além de questionarem os instrutores com as suas dúvidas.

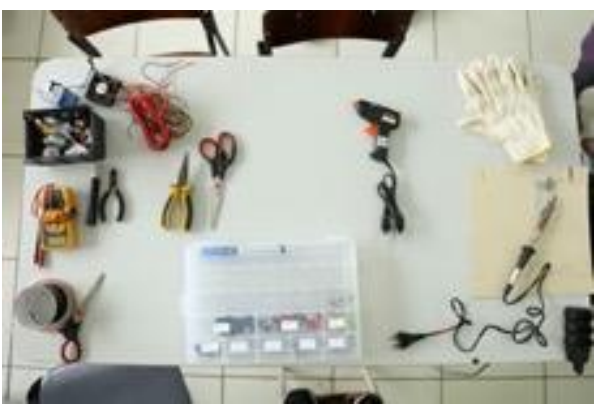

Figura 6 - Bancada da eletrônica

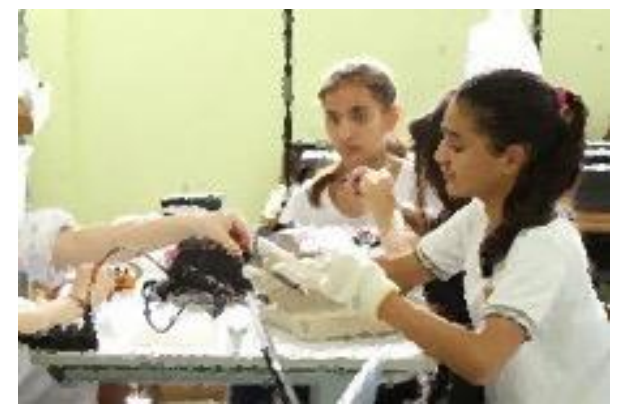

Figura 7 - Estudantes soldando 
V Congresso Brasileiro de Informática na Educação (CBIE 2016)

Anais do XXII Workshop de Informática na Escola (WIE 2016)

- Bancada Papercraft. A bancada da Plotter de Corte, a Silhouette ${ }^{7}$ e o Silhouette Studio foram apresentados aos estudantes. Foi explicado como a Plotter funcionava através de seus 2 (dois) eixos e a lâmina, além de demonstrar alguns exemplos de uso. Para demonstrar a Silhouette em funcionamento foi explicado aos estudantes uma base sobre gramatura de papel e as possíveis aplicações do vinil adesivo, cada estudante pode então escolher uma imagem utilizando serviços de busca na internet, para então manipulá-la no Silhouette Studio e recortar seu próprio adesivo personalizado.

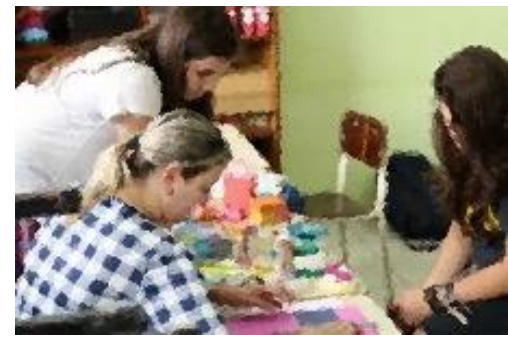

Figura 8 - Estudantes nabancada de Papercraft

No segundo dia de projeto, os alunos iniciaram o planejamento e o desenvolvimento dos seus projetos. Neste segundo contato foi explicado aos estudantes o conceito de planejamento de projeto, incentivando-os a planejar antes de iniciar os trabalhos. A atividade de planejamento dos projetos tomou em torno de 1 (uma) hora, restando nesse dia 3 (três) horas para execução.

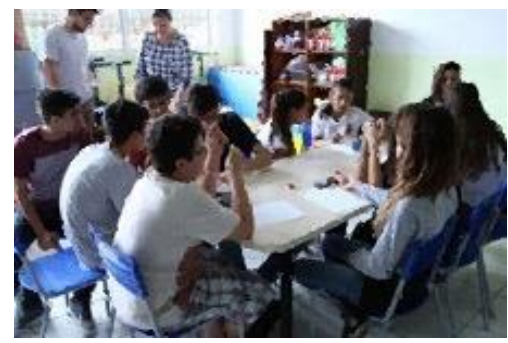

Figura 9 - Estudantes planejando os projetos

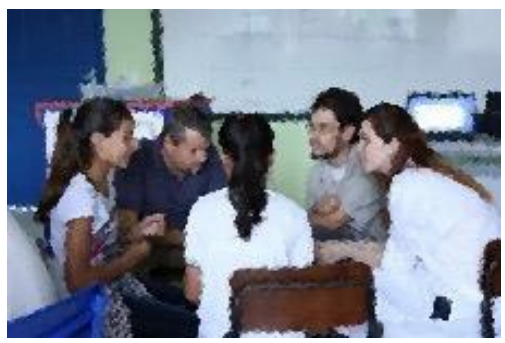

Figura 10 - Estudantes sanando dúvidas

Após os estudantes planejarem e sanarem suas dúvidas, com os colaboradores/facilitadores, iniciou-se a execução do que foi planejado, intercalando as bancadas de acordo com as suas necessidades. Nesta fase, identificou-se a necessidade de algum método de controle do uso das bancadas, através de experiências anteriores, foi decidido por utilizar o método Kanban - para controlar o fluxo da produção, porém sem fichas. Foi utilizado o quadro negro para sinalizar qual bancada estava utilizando por cada equipe, também se percebeu que algumas equipes tinham mais afinidade ou interesse em uma determinada bancada do que em outra, fato que se deu de acordo com o perfil de cada uma.

No terceiro dia os estudantes deram continuidade aos projetos iniciados no encontro anterior, os estudantes focaram em adequar o mais rápido possível e em dar acabamento nas suas propostas a fim de apresentá-las, foi um dia para resolver imprevistos nas partes finais dos projetos, as 4 (quatro) equipes apresentaram seus projetos no horário estipulado.

\footnotetext{
${ }^{7}$ www.silhouettebrasil.com.br
} 
V Congresso Brasileiro de Informática na Educação (CBIE 2016)

Anais do XXII Workshop de Informática na Escola (WIE 2016)

Uma das equipes teve a finalidade de projetar um moinho de água que gerasse energia o suficiente para acender os leds, porém precisaram readequar o projeto para que pudesse ser finalizado a tempo e optaram por fazer uma versão menos funcional e mais estética com um cata-ventos de papel, leds em série alimentados por uma bateria e um suporte de madeira (Figura 11).

O projeto apresentado na Figura 12 desenvolveu 3 (três) caixas, uma com um Led alimentado por bateria e um adorno em forma de âncora (impresso na impressora 3D) que reproduziu um "baú do tesouro" e outras 2 (duas) caixas semelhantes a um porta canetas com algumas tags reproduzidas na bancada de Papercraft.

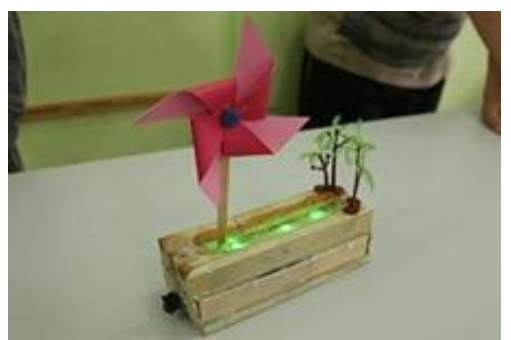

Figura 11 - Projeto de um Cata-ventos

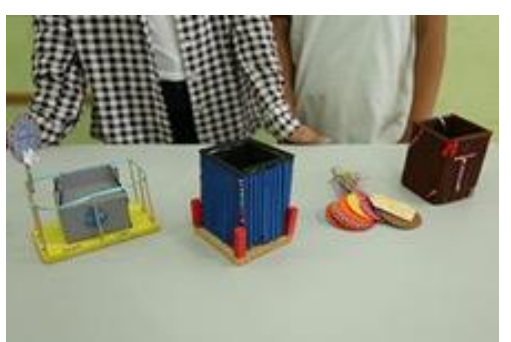

Figura 12 - Projeto do Baú do tesouro

A Figura 13 apresenta o projeto do trabalho de restauração em caixas, que a equipe já possuía, porém deram um novo acabamento de superficie e reproduziram algumas tags disponíveis na bancada de Papercraft.

Outra equipe projetou uma representação de uma casa com materiais da bancada de marcenaria e com iluminação interna com leds e um interruptor para o mesmo (Figura 14).

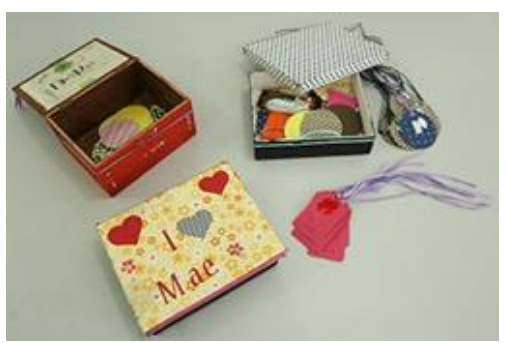

Figura 13 - Projeto de restauração

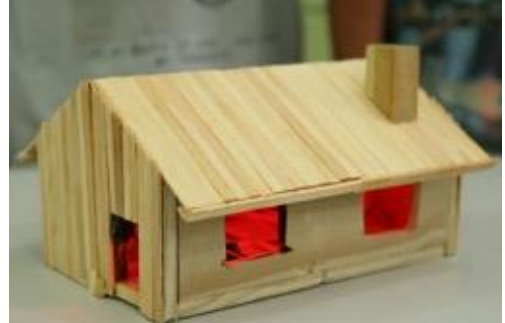

Figura 14 - Projeto de representação de uma casa

Nesse último dia, o período de trabalho foi diminuído para que os estudantes pudessem apresentá-los e para que fosse realizado um debate em grupo com todos os estudantes e os próprios professores da E.B. Yolanda Laurindo Ardigó, com a finalidade de averiguar quais os conhecimentos os estudantes tinham absorvido desta experiência e se as equipes tinham atingido todos os objetivos planejados para os seus projetos.

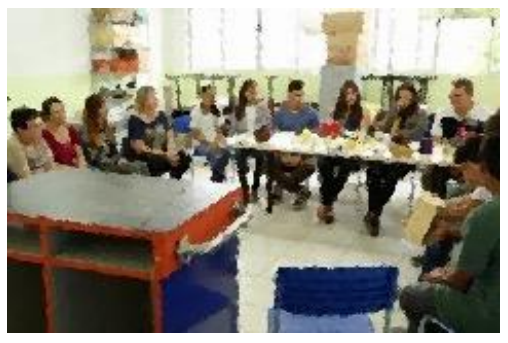

Figura 15 - Debate em grupo

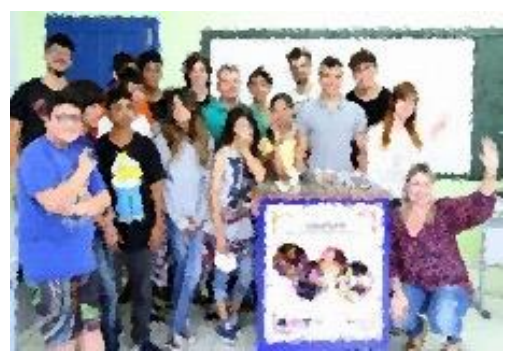

Figura 16 - Participantes das atividades praticas 
V Congresso Brasileiro de Informática na Educação (CBIE 2016)

Anais do XXII Workshop de Informática na Escola (WIE 2016)

Nas atividades realizadas com as diversas bancadas não houve a participação direta dos professores da escola básica, pois as atividades foram realizadas no contra turno das aulas regulares dos estudantes. Porém, diferentes núcleos da escola e da comunidade escolar colaboraram com o planejamento, o andamento e a divulgação deste projeto. Fato que apoiou a participação dos estudantes que se envolveram e mostraram interesse pelas atividades aplicadas.

Durante o desenvolvimento dos projetos, observou-se que a bancada de marcenaria deveria estar fora do ambiente de criação. $O$ barulho, cheiro, poeira, serragem que a bancada gera, criam desconforto para os envolvidos nos demais projetos que estavam sendo desenvolvidos no mesmo espaço físico.

A estrutura do Lite Maker que foi utilizada como base para a bancada de marcenaria, mostrou-se ser instável. Não oferecendo estabilidade necessária para a execução das atividades pertinentes a essa bancada.

\section{Conclusões}

Os estudantes apresentaram interesse em especial em conhecer a impressora 3D. O leque de possibilidades dessa tecnologia despertou a curiosidade de seus observadores, que buscam desvendar o seu funcionamento. A tecnologia embutida na impressora faz com que ela seja um produto com um nível de complexidade elevado, o que aguça a curiosidade dos jovens estudantes.

$\mathrm{O}$ uso de impressoras $3 \mathrm{D}$ viabiliza uma série de aspectos positivos relacionado a prototipação de um produto, entretanto, após a experiência realizada foi possível verificar que não é um equipamento imprescindível para as atividades, uma vez que o tempo de execução não atende as demandas dos estudantes com a mesma eficiência dos outros equipamentos. Vale ressaltar que o uso de equipamentos que priorizam as práticas manuais teve mais atenção, em questão de frequência de uso, por parte dos estudantes. A possibilidade de remover a impressora 3D do Lite Maker proporciona redução de custo do equipamento e não gera impactos negativos no desenvolvimento das dinâmicas.

O fator tempo foi um elemento complicador na aplicação das atividades com o Lite Maker. O planejamento das atividades foi realizado no início das mesmas, porém ao decorrer do seu desenvolvimento, muitas vezes, ocorrem alterações e mudanças necessárias demandando novos esforços e tempo que vão além dos especificados inicialmente, fato que prejudica o produto final desenvolvido.

Pontuam-se como resultados positivos da atividade aplicada: o respeito entre os estudantes com relação a diversidade de opinião nos próprios grupos de trabalho e que ocorreu de forma acentuada espírito de equipe. Lite Maker oportunizou aos estudantes a vivência em um ciclo de projeto, passando por etapas de planejamento, execução, pequenas adaptações e avaliação do mesmo.

Todas as bancadas foram utilizadas para o desenvolvimento e criação dos projetos planejados, mesmo sem a obrigatoriedade do uso das mesmas as equipes interagiram entre as bancadas sempre em busca da resolução dos problemas encontrados em seus projetos.

A interação e cooperação entre os estudantes e facilitadores se deram de forma espontânea. Mesmo os alunos que demostravam algum tipo de timidez em aulas 
V Congresso Brasileiro de Informática na Educação (CBIE 2016)

Anais do XXII Workshop de Informática na Escola (WIE 2016)

regulares, durante o desenvolvimento dos projetos Makers se comunicavam de forma objetiva e clara. Um ambiente que disponibiliza recursos para criação associado a facilitadores que conheçam tais recursos transmitem aos estudantes segurança para criação e até mesmo para encarar a resolução dos problemas.

Como resultados positivos vale destacar: o interesse da escola em atividades práticas, a divulgação dos resultados em mídias locais, abertura da escola para integrar atividade em seu plano pedagógico, presença da Gerência de Tecnologias da Secretaria municipal durante os dias de atividades.

Como sugestões para trabalhos futuros sugerem-se tempo mínimo de 30 (trinta) horas de atividades com os estudantes, o uso de mais repositórios e documentos para inspiração dos projetos, dinâmicas de grupo para definir melhor os papéis de cada estudante em sua equipe de trabalho e incluir ferramentas para documentação dos projetos.

\section{Referências}

BERS, M. U; FLANNERY, L.; KAZAKOFF, E. R.; SULLIVAN, A. Computational thinking and tinkering: Exploration of an early childhood robotics curriculum. Computers \& Education, v. 72, p. 145-157, 2014.

Blikstein, P. Digital fabrication and 'making'in education: The democratization of invention. FabLabs: Of machines, makers and inventors", p. 1-21, 2013.

You cannot think about thinking without thinking about what Seymour Papert would think. 2009.

O pensamento computacional e a reinvenção do computador na educação. 2008.

BOMBASAR, James; SANTIAGO, Rafael; MIRANDA, Elisangela M.; RAABE, André L. A. Ferramentas para o Ensino-Aprendizagem do Pensamento Computacional: onde está Alan Turing?. In: Anais do Simpósio Brasileiro de Informática na Educação. 2015. p. 81.

MCKAY, Christian; PEPPLER, Kylie. MakerCart: A Mobile Fab Lab for the Classroom. In: position paper at the Interaction Design for Children Conference (IDC), New York, June. 2013.

Milne, A.; Riecke, B.; Antle, A. Exploring Maker Practice: Common Attitudes, Habits and Skills from Vancouver's Maker Community. Studies, v. 19, n. 21, 2014. p. 23.

PAPERT, S. Mindstorms: Children, computers and powerful ideas. Basic Books, inc. Artes Médicas: Porto Alegre. 1980.

SANTANA, A. L. M. Análise do processo metodológico de montagem de um brinquedo de programar. 2015. 93 f. Dissertação (Mestrado) - Curso de Computação Aplicada, Universidade do Vale de Itajaí, Itajaí, 2015. 